\title{
Advanced urethral paraganglioma treated with axitinib; outcome and comprehensive molecular analysis
}

\begin{abstract}
Introduction: Paraganglioma is a rare entity that arises from extra-adrenal paraganglia and accounts for less than a quarter of all chromaffin cell-related tumors. There are few cases of urethral paragangliomas reported on the literature. Most of them are hormonally inactive and local excision is curative in localized disease. We present a metastatic urethral paraganglioma in 71 years-old man that underwent a comprehensive search for molecular alterations amenable to pharmacological targeting.
\end{abstract}

Materials and Methods: The following molecular studies were performed in tumor tissue: HER-2/neu amplification, c-Kit immunohistochemistry, EGFR and BRAF mutations and Foundation MedicineT5a panel (next generation sequencing); in peripheral blood germ line alterations in genes related to familiar paraganglioma were also analyzed.

Results: Though no genetic alteration was found, the patient achieved tumor control with antiangiogenics (first with sunitinib that was later shifted to axitinib because of drug induced hyperbilirubinemia). Here mains asymptomatic 16 months after initiation of therapy.

Conclusion: Paraganglioma should be considered as a tumor constitutively addictive to angiogenesis even in the absence of pathogenic mutations or rearrangements in such pathway.
Volume 2 Issue 2 - 2015

\author{
Chen X,' Doger B, Rodriguez-Moreno JF,' \\ Romero N,' Garcia-Donas J' \\ 'Clara Campal Comprehensive Cancer Center, Spain \\ ${ }^{2}$ Universitary Hospital Infanta Cristina, Spain
}
Correspondence: Xin Chen, Clara Campal Comprehensive Cancer Center; Ona street 10 (Madrid), Madrid, postal (zip) code 28050, Spain, Tel 34917567800,

Email xchen@hmhospitales.com

Received: January 14, 2014 | Published: February 28, 2015
Abbreviations: 131I-MIBG, 131I-meta iodo benzyl guanidine scintigraphy; MIBG, meta iodo benzyl guanidine; SDHB, succinate de hydrogenase subunit B gene

\section{Case presentation}

A 71 year-old-man, with Gilbert's syndrome as the only medical history, presented in February 2013 recurrent episodes of urinary retention and haematuria. Initial diagnosis was benign hyperplasia of the prostate thus $0.4 \mathrm{mg}$ of tamsulosin hydrochloride once a day was prescribed. Symptoms did not improve and four months later a retro pubic adenectomy was performed. Pathological report described a 4.5 $\mathrm{cm}$ length malignant tumor of the prostatic urethra with an immunophenotype compatible with paraganglioma (positive chromogranin, synaptophysin and S100; negative CK AE1/AE3, EMA, CK 7, CK 20 and CD10); Ki67 index was 5\% and the tumor affected surgical margins.

A body CT scan revealed multiple pelvic adenopathies and implants as well as thickening of the urinary bladder wall. A PETCT with 18F-FDG confirmed such findings (Figure 1A \& 1B). Urinary and serous catecholamines were in normal range and 131I-metaiodobenzylguanidine (131I-MIBG) scintigraphy did not show any uptake. Since this is an infrequent condition where little therapeutic options are available, a comprehensive molecular study was initiated aiming to identify alterations amenable to pharmacological targeting. FISH for HER-2/neu gene amplification and ALK translocations, immunohistochemical staining for c-Kit and sequencing of hotspot mutations in the EGFR gene (by the Cobas $^{\circledR}$ EGFR Mutation Test) and BRAF gene $\left(\right.$ Cobas $^{\circledR} 4800$ BRAF V600 Mutation Test) were performed showing no alteration.

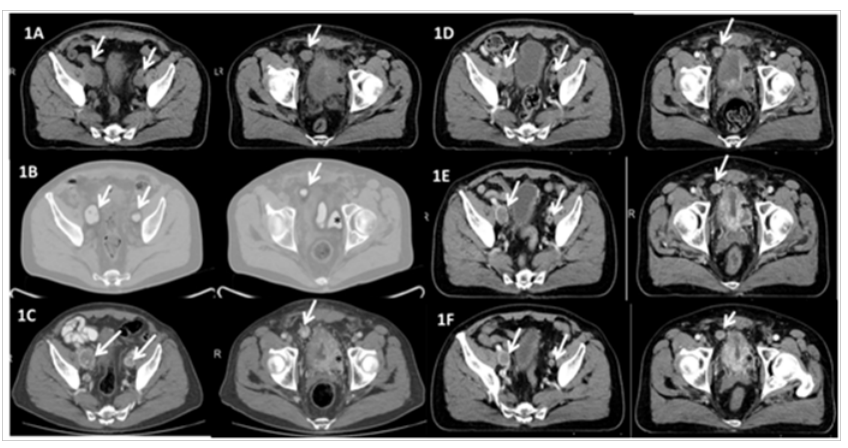

Figure I Radiological assessments at diagnosis and along treatment. Left: pelvic adenopathies (white arrows); Right: Tumoral thickening of the urinary bladder wall (black asterisk) and pelvic implant (white arrow)

IA Baseline CT scan (July 20I3)

IB Baseline PET CT scan (July 20I3)

IC CT scan (September 20I3) after two cycles of cisplatin+etoposide. Enlargement of pelvic nodes and bladder mass compared to baseline ID CT scan (December 20I3) after 2 months on sunitinib IE CT scan (July 20I4) after 3 months on axitinib IF CT scan (October 20I4) after 7 months on axitinib.

Additionally tumor samples were subjected to Foundation Medicine T5a test, an assay based on massively parallel DNA sequencing designed to characterize base substitutions, short insertions and deletions (indels), copy number alterations and selected fusions across 287 cancer-related genes. No relevant findings were made (see extra material for a detailed description of the assay, (Table 1)). 
Finally germ line DNA was studied through sequencing and multiplex ligation-dependent probe amplification (MLPA) of genes previously related with hereditary paraganglioma (VHL, SDHA, B, C and D, SDHF1 and 2 and MAX). No pathogenic alteration was identified (Table 2). In July 2013 the patient started first line chemotherapy with cisplatin $30 \mathrm{mg} / \mathrm{m}^{2}$ and etoposide $100 \mathrm{mg} / \mathrm{m}^{2}$ days 1 to 3 every 21 days. Toxicity included grade II nausea, grade III constipation, grade III neutropenia and dysgeusia. After two cycles radiological progression was observed and tumor related symptoms worsened (Figure 1C). In October 2013 the patient started sunitinib (37.5 mg daily) with quick improvement of urinary symptoms. He developed conjunctival jaundice (due to grade II hyperbilirubinemia, total bilirubin up to 2.89 $\mathrm{mg} / \mathrm{dl}$ ), grade II hypertension, grade II hand-foot syndrome and grade II hypothyroidism that required hormonal replacement, leading to a dose reduction to $25 \mathrm{mg}$ daily. After two months on therapy, CT scan showed stable disease by RECIST criteria with a decrease in size of some pelvic adenopathies (Figure 1D). No liver metastasis was observed.

Side effects were properly controlled but hyperbilirubinemia, which rose again up to $3.38 \mathrm{mg} / \mathrm{dl}$. Treatment was held and reintroduced at an intermittent schedule (daily sunitinib $25 \mathrm{mg}, 3$ weeks on, one week off). After six months on treatment stable disease was confirmed in CT scan but sunitinib was definitely discontinued because of recurrent hyperbilirubinemia. On April 2014 the patient started axitinib 5mg every 12 hours but a dose reduction to $5 \mathrm{mg}$ daily was again required because of hyperbilirubinemia (serum bilirubin $2.88 \mathrm{mg} / \mathrm{dl}$ ). Currently the patient remains asymptomatic and on treatment, 16 months after initiation of antiangiogenics (Figure 1E \& 1F).

Table I List of 287 genes studied in the Foundation Medicine T5a assay

\begin{tabular}{|c|c|c|c|c|c|c|c|c|}
\hline$A B L I$ & GID4 & CUL4B & FGF23 & IRF4 & MSH6 & PDGFRA & RUNXI & WISP3 \\
\hline AKTI & CARDII & CYPI7AI & FGF3 & IRS2 & MTOR & PDGFRB & RUNXITI & WTI \\
\hline AKT2 & CASP8 & DAXX & FGF4 & JAKI & MUTYH & PDKI & SETD2 & WTX \\
\hline AKT3 & CBFB & DDR2 & FGF6 & JAK2 & MYC & PIK3C2G & SF3BI & XPOI \\
\hline ALK & CBL & DIS3 & FGF7 & JAK3 & MYCLI & PIK3C3 & $\mathrm{SH} 2 \mathrm{~B} 3$ & $X R C C 3$ \\
\hline ALOXI2B & CCNDI & DNMT3A & FGFRI & JUN & MYCN & PIK $3 C A$ & SMAD2 & ZNF217 \\
\hline APC & CCND2 & DOTIL & FGFR2 & KDM5A & MYD88 & PIK3CG & SMAD4 & ZNF703 \\
\hline APCDDI & CCND3 & EGFR & FGFR3 & KDM5C & MYST3 & PIK3RI & SMARCA4 & \\
\hline $\mathrm{AR}$ & CCNEI & EMSY & FGFR4 & KDM6A & NBN & PIK3R2 & SMARCBI & \\
\hline ARAF & CD79A & EP300 & FLTI & KDR & NCORI & PMS2 & SMARCDI & \\
\hline ARFRPI & CD79B & EPHA3 & FLT3 & KEAPI & NFI & PNRCI & SMO & \\
\hline ARIDIA & CDC73 & EPHA5 & FLT4 & $\mathrm{KIT}$ & NF2 & PPP2RIA & SOCSI & \\
\hline ARID2 & $\mathrm{CDHI}$ & EPHBI & FOXL2 & KLHL6 & NFE2L2 & PRDMI & SOXIO & \\
\hline ASXLI & CDK 12 & ERBB2 & GATAI & KRAS & NFKBIA & PRKARIA & sox2 & \\
\hline ATM & CDK4 & ERBB3 & GATA2 & LMOI & NKX2-I & PRKDC & SPEN & \\
\hline ATR & CDK6 & ERBB4 & GATA3 & LRPIB & NOTCHI & PRSS8 & SPOP & \\
\hline ATRX & CDK8 & ERG & GNAII & MAP2KI & $\mathrm{NOTCH} 2$ & PTCHI & SRC & \\
\hline AURKA & CDKNIB & ESRI & GNAI3 & MAP2K2 & NOTCH3 & PTEN & STAG2 & \\
\hline AURKB & CDKN2A & $\mathrm{EZH} 2$ & GNAQ & MAP2K 4 & $\mathrm{NOTCH} 4$ & PTPNII & STAT4 & \\
\hline$A X L$ & CDKN2B & FAM46C & GNAS & MAP3KI & NPMI & RAD50 & STKII & \\
\hline $\mathrm{BACHI}$ & CDKN2C & FANCA & GPRI 24 & MAP3KI 3 & NRAS & RAD5I & SUFU & \\
\hline BAPI & CEBPA & FANCC & GRIN2A & MCLI & NSDI & RAD5IB & SYK & \\
\hline BARDI & CHEKI & FANCD2 & GSK3B & MDM2 & NTRKI & RAD5IC & TBX3 & \\
\hline $\mathrm{BCL2}$ & CHEK2 & FANCE & HGF & MDM4 & NTRK2 & RAD5ID & TET2 & \\
\hline BCL2L2 & CHUK & FANCF & HLA-A & MED 12 & NTRK3 & RAD52 & TGFBR2 & \\
\hline BCL6 & $\mathrm{ClC}$ & FANCG & HRAS & MEF2B & NUP93 & RAD54L & TIPARP & \\
\hline BCOR & CRBN & $\mathrm{FANCl}$ & IDHI & MENI & PAK3 & RAFI & TNFAIP3 & \\
\hline BCORLI & CREBBP & FANCL & IDH2 & MET & PAK7 & RARA & TNFRSFI4 & \\
\hline BLM & CRKL & FANCM & IGFI & MITF & PALB2 & RBI & TOPI & \\
\hline BRAF & CRLF2 & FAT3 & IGFIR & MLHI & PARPI & REL & TP53 & \\
\hline BRCAI & CSFIR & FBXW7 & IGF2 & MLL & PARP2 & RET & TRRAP & \\
\hline BRCA2 & $\mathrm{CTCF}$ & FGFIO & IKBKE & MLL2 & PARP3 & RICTOR & $\mathrm{TSCl}$ & \\
\hline BRIPI & CTNNAI & FGFI2 & IKZFI & MPL & PARP4 & RNF43 & TSC2 & \\
\hline BTGI & CTNNBI & FGFI4 & IL7R & MREIIA & PAX5 & RPAI & TSHR & \\
\hline BTK & CUL4A & FGFI9 & INHBA & $\mathrm{MSH} 2$ & PBRMI & RPTOR & VHL & \\
\hline \multicolumn{9}{|c|}{ Rearrangements } \\
\hline ALK & BRAF & ETV4 & EWSRI & NTRKI & RARA & TMPRSS2 & & \\
\hline $\mathrm{BCL} 2$ & EGFR & ETV5 & MLL & PDGFRA & RET & & & \\
\hline $\mathrm{BCR}$ & ETVI & ETV6 & MYC & RAFI & ROSI & & & \\
\hline
\end{tabular}


Table 2 Description of direct sequencing and Multiplex Ligation-dependent Probe

\begin{tabular}{|c|c|c|}
\hline Gene & $\begin{array}{l}\text { Molecular } \\
\text { study }\end{array}$ & Technic \\
\hline \multirow{4}{*}{ SDHA } & \multirow{4}{*}{ sequencing } & PCR and direct sequencing was performed of the coding region and flanking intronic region (8bp) of the genes: \\
\hline & & SDHA (reference sequence NM_004I68.2*; chromosome 5) \\
\hline & & SDHB (reference sequence NM_003000.2; chromosome I) \\
\hline & & SDHC (reference sequence NM_00300I.3; chromosome I) \\
\hline \multirow{2}{*}{ SDHB } & \multirow{2}{*}{ sequencing } & SDHD (reference sequence NM_003002.2; chromosome II) \\
\hline & & VHL (reference sequence NM_00055I.3; chromosome 3) \\
\hline SDHC & sequencing & $\begin{array}{l}\text { Capture target regions using oligonucleotide probes (Next era rapid, llumina) and subsequent realization of next } \\
\text { generation sequencing (Miseq, llumina) was completed. The alignment and identification of bases were performed using } \\
\text { the Burrows / Wheeler Aligher, BWA Miseq reporter), followed by analysis with Next Gene (Soft Genetics) program. } \\
\text { When necessary, the Sanger sequencing was performed in the regions in which bases were insufficient cover aged. } \\
\text { average coverage lower than I00X or minimum coverage lower than 20X * }\end{array}$ \\
\hline SDHC & sequencing & \\
\hline \multirow[t]{2}{*}{ VHL } & sequencing & \\
\hline & \multirow{9}{*}{$\begin{array}{l}\text { deletions / } \\
\text { duplications }\end{array}$} & MLPA analysis (MRC Holland ) of the chromosomal region comprising the genes: \\
\hline SDHA & & SDHA (cr5) Reference sequence NM_004I68.2 (SDHA) \\
\hline SDHB & & SDHB (crl) Reference sequence NM_003000.2 (SDHB) \\
\hline SDHC & & SDHC (crl) Reference sequence NM_00300I,3 (SDHC) \\
\hline SDHD & & SDHD (crl I) Reference sequence NM_003002,2 (SDHD) \\
\hline SDHAFI & & SDHAFI (cr 19) Reference sequence NM_00I04263I.2 (SDHAFI) \\
\hline SDHAF2 & & SDHAF2 (cr II) Reference sequence NM_0I784I.2 (SDHAF2) \\
\hline VHL & & VHL (cr3) Reference sequence NM_00055I.3. (VHL) \\
\hline MAX & & MAX (cr I4) Reference sequence NM_002382,4 (MAX) \\
\hline
\end{tabular}

Amplification performed on germ line DNA.

\section{Discussion}

Paraganglioma is an infrequent tumor that arises from extraadrenal paraganglia and accounts for less than a quarter of cases of all chromaffin cell-related tumors. There are few cases of urethral paragangliomas reported on the literature. Most of these tumors are hormonally inactive. Although haematuria may be the presenting symptom, it is important to exclude additional more common and possibly more sinister lesions such as transitional cell carcinoma. Local excision appears to be curative in most of reported cases. $^{1,2}$

Surgery and metaiodobenzylguanidine (MBIG) are cornerstones of treatment for advanced disease. ${ }^{3,4}$ Unfortunately our case was deemed as unresectable due to multiple pelvic implants and no MIBG uptake was observed at diagnosis. Chemotherapy was initially administered but demonstrated to be useless and toxic. A comprehensive search for molecular alterations amenable to pharmacological targeting failed to guide treatment choice thus; antangiogenic therapy was initiated based on recently communicated data with sunitinib. ${ }^{5-9}$ Though tumor control and clinical improvement were achieved, recurrent hyperbilirubinemia, likely related to Gilbert's syndrome led to a switch to axitinib. Recently a link between this syndrome and hyperbilirubinemia along sunitinib or pazopanib treatment has been shown. This is the first report to communicate clinical benefit of a malignant paraganglioma treated with axitinib.

Around $50 \%$ of metastatic paraganglioma is caused by hereditary germ line mutations of the mitochondrial enzymatic complex II succinate dehydrogenase subunit B gene (SDHB) which finally produces a downstream activation of angiogenesis. Additionally vascular endothelial growth factors and their receptors 1 and 2 are known to be over expressed in metastatic pheocromocitomas and paragangliomas regardless of SDHB mutations. ${ }^{10}$

Other genes involved in hereditary paragangliomas (VHL, SDHA, $\mathrm{C}$ and $\mathrm{D}$ and their cofactors SDHAF and MAX) are known to cause a similar stimulation of angiogenesis. These findings have led to the design of two clinical trials assessing the utility of both sunitinib and axitinib in pheochromocitoma and paraganglioma (NCT00843037 and NCT01967576, respectively). ${ }^{11}$ Interestingly our case, despite a comprehensive molecular analysis, did not present alterations in neither the mentioned genes nor additional 287 cancer related genes included in the Foundation Medicine T5a test. ${ }^{12}$ These studies cover all the recommended genes regarding screening of familiar paraganglioma. ${ }^{13}$ However we did not study epigenetic alterations that have been described as major contributors to some related pathologies as renal carcinoma, nor mutations in any additional genes. Thus, activators of angiogenesis could be present in the tumor but undetected by our techniques.

\section{Conclusion}

Paraganglioma should be considered as a tumor constitutively addictive to angiogenesis even in the absence of pathogenic mutations or rearrangements. 


\section{Acknowledgments}

None.

\section{Conflicts of interest}

Authors declare that there is no conflict of interest.

\section{References}

1. Boyle M, Gaffney EF, Thurston A. Paraganglioma of the prostatic urethra. A report of three cases and a review of the literature. Br J Urol. 1996;77(3):445-448.

2. Chen J, Quan CY, Jiang N, et al. Paraganglioma of the prostate: a case report and review of the literature. Zhonghua Nan Ke Xue. 2012;18(8):715-718.

3. NCCN Guidelines Version 2: Neuro endocrine tumors. NCCN Washington, USA; 2014. 1-99 p.

4. Plouin PF, Fitzgerald P, Rich T, et al. Metastatic Pheochromocytoma and Paraganglioma: Focus on Therapeutics. Horm Metab Res. 2012;44(5):390-399.

5. Prochilo T, Savelli G, Bertocchi P, et al. Targeting VEGF-VEGFR Pathway by Sunitinib in Peripheral Primitive Neuroectodermal Tumor, Paraganglioma and Epithelioid hemangio endothelioma: Three Case Reports. Case Rep Oncol. 2013;6(1):90-97.
6. Cirillo F. Metastatic Paraganglioma and treatment with sunitinib: a case report. Tumori. 2010;96(6):1022-1077.

7. Hahn NM, Reckova M, Cheng L, et al. Patient with malignant paraganglioma responding to the multikinase inhibitor sunitinib malate. J Clin Oncol. 2009;27(3):460-463.

8. Jimenez C, Cabanillas ME, Santarpia L, et al. Use of the tyrosine kinase inhibitor sunitinib in a patient with von Hippel-Lindau disease: targeting angiogenic factors in pheochromocytoma and other von Hippel-Lindau disease-related tumors. J Clin Endocrinol Metab. 2009;94(2):386-391.

9. Ayala-Ramirez M, Chougnet CN, Habra MA, et al. Treatment with Sunitinib for Patients with Progressive Metastatic Pheochromocytomas and Sympathetic Paragangliomas. $J$ Clin Endocrinol Metab. 2012;97(11):4040-4050.

10. Jimenez C, Rohren E, Habra MA, et al. Current and Future Treatments for Malignant Pheochromocytoma and Sympathetic Paraganglioma. Curr Oncol Rep. 2013;15(4):356-371.

11. clinicaltrials.gov, visited on November 2014.

12. Frampton GM, Fichtenholtz A, Otto GA, et al. Development and validation of a clinical cancer genomic profiling test based on massively parallel DNA sequencing. Nat Biotechnol. 2013;31(11):1023-3101.

13. Cascon A, Pita G, Burnichon N, et al. Genetics of Pheochromocytoma and Paraganglioma in Spanish Patients. J Clin Endocrinol Metab. 2009;94(5):1701-1705. 